\title{
SCORPION ENVENOMATION SYMPTOMS IN PREGNANT WOMEN
}

\section{BEN NASR H. (1), HAMMAMI T. S.(2), SAHNOUN Z. (2), REBAI T. (3), BOUAZIZ}

M. (4), KASSIS M. (5), ZEGHAL K. M. (2)

(1) Department of Biology, Bizerte Faculty of Sciences, University of $7^{\text {th }}$ November at Carthage, Tunis, Tunisia; (2) Laboratory of Pharmacology, Sfax Faculty of Medicine, University of Sfax, Sfax, Tunisia; (3) Laboratory of Embryology and Histology, Sfax Faculty of Medicine, University of Sfax, Sfax, Tunisia; (4) Reanimation Service, Habib Bourguiba Hospital, Sfax, Tunisia; (5) Community Medicine and Epidemiological Service, Hédi Chaker Hopital, Sfax, Tunisia.

ABSTRACT: Scorpion envenomation is common in many countries; however, its effects on pregnancy are still unclear. In the present paper, we described the effects of scorpion envenomation on pregnant patients. A retrospective study was carried out considering the clinical and laboratory exams of patients admitted to the emergency room of Habib Bourguiba Hospital, Sfax, Tunisia, from 1990 to 2004. Variability of these clinical and laboratory profiles according to maternal age, gestational age and number of previous parities was also discussed. Among 167 scorpion-envenomed women, age ranged from 17 to 42 years, $7.18 \%$ were pregnant. These presented symptoms similar to those of non-pregnant women envenomed by scorpions. Two pregnant patients developed intense pelvic pain and one manifested vaginal bleeding. Although the studied parameters showed non-significant differences, we could conclude that scorpion envenomation may lead to abnormal uterine contraction probably causing preterm delivery. Maternal disturbances induced by scorpion envenomation may influence the fetus development. The effects were more severe in the second trimester of pregnancy.

KEY WORDS: pregnant women, scorpion envenomations, signs and symptoms, laboratory data.

CONFLICTS OF INTEREST: There is no conflict.

\section{CORRESPONDENCE TO:}

BEN NASR HMED, Laboratoire de Pharmacologie, Faculté de Médecine de Sfax, Université de Sfax; Rue Magida Boulila, 3029, Sfax, Tunisia. Phone: 21674247036. Fax: 2177424 6217. Email: hamdoscie@voila.fr. 


\section{INTRODUCTION}

Scorpion envenomation is a common occurrence in tropical and sub-tropical countries (7). Its clinical manifestations are largely varied, and its pathophysiology essentially includes nervous $(17)$, cardiovascular $(17,32)$ and muscular systems disturbances. Little is known about the effect of scorpion envenomation on human pregnancy (16). In the present study, we described the signs and symptoms of scorpion-envenomed pregnant women based on a retrospective study.

\section{PATIENTS AND METHODS}

The emergency room of the Habib Bourguiba Hospital, Sfax, Tunisia, manages severe cases of scorpion envenomation, including those of grade II: hyperthermia, shivering, agitation, hypertension, tachycardia, etc.; and the ones of grade III: coma, cardiovascular arrest, etc. (14). Most of these patients are from the southern regions of Tunisia.

A retrospective study about the laboratory data of scorpion-envenomed pregnant patients admitted to Habib Bourguiba Hospital from 1990 to 2004 was carried out.

Twelve pregnant women (7.18\%) out of one hundred and sixty-seven scorpionenvenomed patients at reproductive age were evaluated. All patients presented primiparous gestation.

Treatments such as scarification and chlorination, previously administered to the patients, and the implicated scorpion species (unidentified) were ignored in this study. All patients had received a combination of the following treatments: antiscorpion sera, corticoids, phenobarbital, diazepam, hydrocortisone hemisuccinate, salbutamol and oxygenation. The time elapsed between the scorpion sting and the evaluation at the hospital emergency room ranged from 50 minutes to 6 hours and 45 minutes. Variability of the patients' clinical and laboratory profiles according to maternal age, gestational age and number of previous parities was analyzed. Data from eight non-pregnant but envenomed women were randomly chosen to compare clinical and laboratory profiles. Data were analyzed using Anova and Kruskal-Walis tests. 


\section{RESULTS AND DISCUSSION}

Neither maternal or fetal death, nor preterm fetal delivery was observed among the twelve scorpion-envenomed pregnant patients studied. Ultrasonographic exams revealed no abnormalities either in the fetuses or in the placentas.

The majority of the pregnant patients showed typical symptoms of scorpion envenomation like pronounced hypertension (66.66\%), tachycardia (58.33\%), agitation (50\%) and increased respiratory rate (50\%) (Figure 1), which corroborated the observations reported by Ismail (10). Two pregnant patients (16.66\%) manifested intense pelvic pain. One of the patients (8.33\%), in her second trimester of pregnancy, showed vaginal bleeding after scorpion sting, which disappeared before hospitalization (Table 1). Intense pelvic pain and hypertension were also noticed in pregnant women envenomed by the black widow spider $(27,29)$, and according to Sherman et al. (29), such signs are similar to those observed in preeclampsia. Milson et al. (20) suggested that intense pelvic pain might result from abnormal uterine contractions. Therefore, these symptoms suggested that scorpion venom may lead to abnormal uterine contractions in pregnant women.

Different scorpion species are usually found at Tunisia region. Among the species most frequently involved in severe envenomation cases are Buthus occitanus tunetanus, Androctonus australis and Androctonus aeneas (11). Like other OldWorld species, their venoms contain numerous neurotoxins that affect the activity of many ion channels of excitable cells. Such actions may be responsible for the contractions of non-pregnant female uteri $(18,19,21,22)$ induced by the venom of different Old-World scorpion species. Scorpion venom has increased estradiol serum levels (21), and prostaglandin F2 alpha uterine biosynthesis (1) may also increase uterine contraction via its bradykinin-potentiating fraction. Such contractions may have been responsible for the pelvic pain noticed in the present study, although specific tests such as electrophysiological exams were not carried out.

Many factors, such as preterm delivery, perinatal morbidity and mortality (23), have been associated with increased metrorrhagia in the second trimester of gestation. Vaginal bleeding, probably indicating pregnancy loss, occurred in one out of the twelve cases studied (8.33\%). This rate is greater than that of preterm labor in Tunisian pregnant women (10\%o - source not cited). Thus, a venom-induced abortion could be concluded. 
No significant variability of clinical and laboratory profiles according to the studied parameters was found (Table 2). The undefined scorpion species, the patients' body weight, and the individual differences, as well as previous treatments, are important factors implicated in the vulnerability to envenomation $(5,12,13)$. An increase in mean arterial pressure, heart rate, glucose level and number of white blood cells was observed in scorpion-envenomed pregnant women compared with the nonpregnant ones. These signs should be taken into consideration and may require different treatments.

Increased white blood cell count, frequently observed in scorpion-envenomed humans, may increase the serum levels of inflammatory and pre-inflammatory kinins and interleukines $(8,9)$. The levels of such compounds in the maternal organism and placental structures have been strongly correlated with uterine rupture, fetal delivery and placental abruption (25), especially in the case of uterine infections and maternal trauma (28). They have been implicated in the regulation and maintenance of the uterine function during pregnancy and parturition $(25,26)$. Increased white blood cell count may also contribute to the increase of histamine levels, which may modify the contractility of the pregnant myometrium (3). Kubow and Koski (15) reported that altered levels of glucose and fatty acids in the maternal organism may retard the fetuses growth. Also, electrolytic disturbances may result in inadequate maternal-fetal exchange of ions and nutrients through the placenta $(4,31)$.

In the present study, the hospitalization period of one pregnant patient was 15 days and that of other three pregnant patients was 4 days, indicating a chronic effect of the scorpion venom on the victims. Thus, scorpion venom can lead to abnormal inutero fetal development via its action on glucose level $(6,10)$, lipid metabolism $(30)$, and electrolyte balance (2). Scorpion-venom-induced respiratory defect (10) in the maternal organism may contribute to the reduction of the inspired oxygen and thereby to fetal hepatic glucose metabolism disturbances, as reported by Parimi et al. (24).

Scorpion envenomation during pregnancy may lead to many biological disturbances influencing the pregnancy process as well as the intrauterine development of the fetus. A systemic experimental study may be helpful to better understand the effects of such envenomation cases, which may influence patient management. 


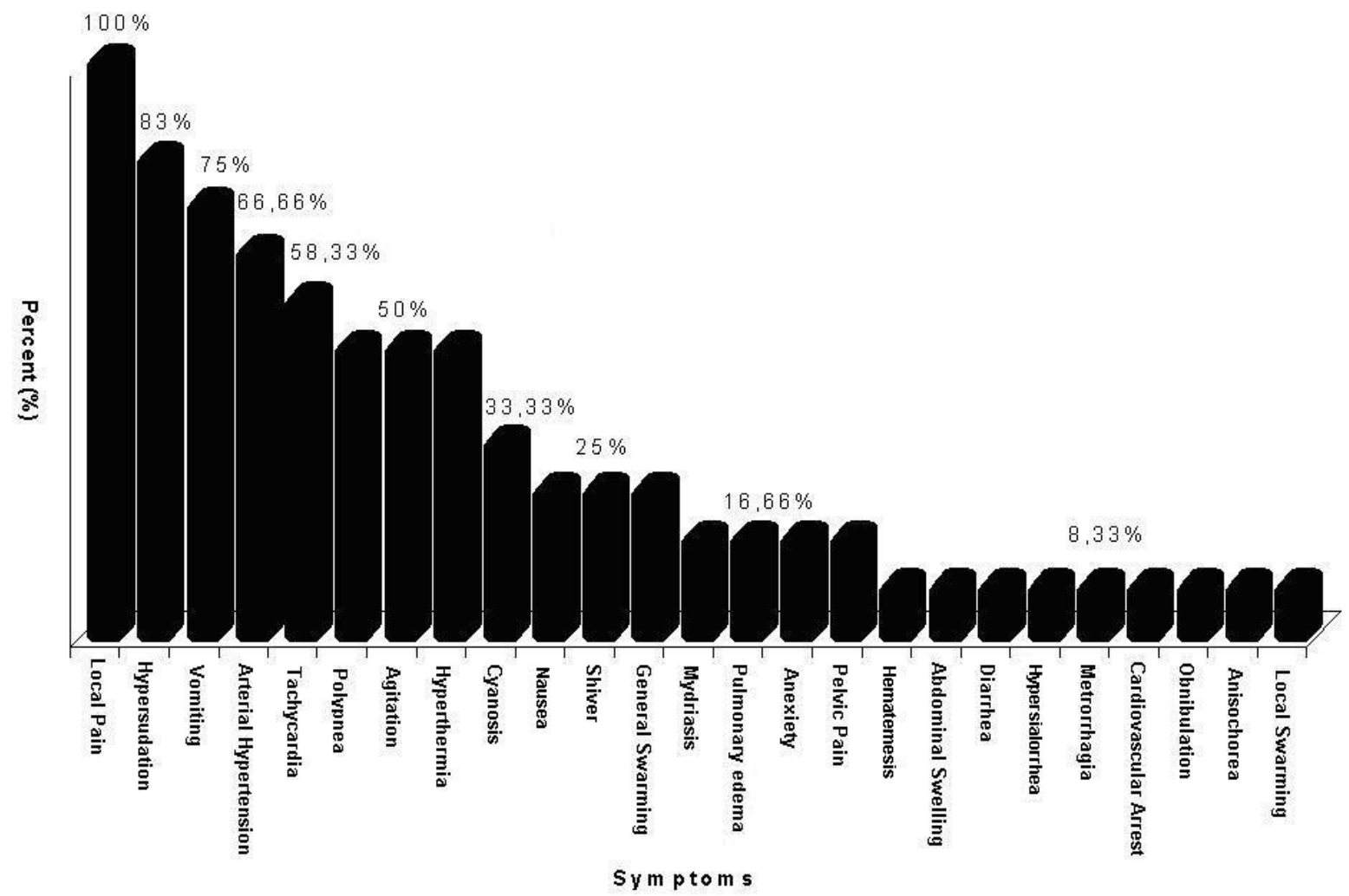

Figure 1 - Percent of symptoms exhibited by pregnant scorpion envenomed women

Table 1: Characterization of pregnant patients envenomed by scorpions.

\begin{tabular}{|c|c|c|c|c|c|c|}
\hline $\begin{array}{l}\text { Patient } \\
\mathrm{N}^{\circ}\end{array}$ & $\begin{array}{c}\text { Age } \\
\text { (years) }\end{array}$ & $\begin{array}{l}\text { Gestational } \\
\text { age }\end{array}$ & $\begin{array}{c}\text { Number of } \\
\text { previous parities }\end{array}$ & Sting site & $\begin{array}{c}\text { Time elapsed } \\
\text { between sting and } \\
\text { medical care (min) }\end{array}$ & $\begin{array}{l}\text { Particular } \\
\text { symptoms }\end{array}$ \\
\hline 1 & 20 & $3^{\text {rd }}$ Trimester & 0 & Leg & 90 & ---- \\
\hline 2 & 22 & $3^{\text {rd }}$ Trimester & 0 & Left hand & 50 & ---- \\
\hline 3 & 22 & $1^{\text {st }}$ Trimester & 0 & Right hand & 120 & ---- \\
\hline 4 & 22 & $1^{\text {st }}$ Trimester & 0 & Right hand & 155 & --- \\
\hline 5 & 22 & $2^{\text {nd }}$ Trimester & 0 & Right foot & 330 & ---- \\
\hline 6 & 23 & $3^{\text {rd }}$ Trimester & 0 & Left foot & 140 & --- \\
\hline 7 & 26 & $1^{\text {st }}$ Trimester & 2 & Right foot & 180 & $\begin{array}{c}\text { Hypertension, } \\
\text { intense pelvic } \\
\text { pain }\end{array}$ \\
\hline 8 & 27 & $2^{\text {nd }}$ Trimester & 0 & Right hand & 240 & ---- \\
\hline 9 & 27 & $2^{\text {nd }}$ Trimester & 2 & The back & 285 & $\begin{array}{c}\text { Hypertension, } \\
\text { intense pelvic } \\
\text { pain, } \\
\text { metrorrhagia }\end{array}$ \\
\hline 10 & 29 & $2^{\text {nd }}$ Trimester & 2 & Left foot & 360 & --- \\
\hline 11 & 40 & $3^{\text {rd }}$ Trimester & 0 & Left foot & 230 & --- \\
\hline 12 & 40 & $2^{\text {nd }}$ Trimester & 3 & Right foot & 225 & ---- \\
\hline
\end{tabular}


Table 2: Variability of clinical and laboratory parameters of scorpion-envenomed patients according to maternal age, gestational age and number of previous parities, and comparison between pregnant and non-pregnant patients envenomed by scorpions.

\begin{tabular}{|c|c|c|c|c|c|c|c|c|c|c|c|}
\hline Parameters & $\begin{array}{c}\text { Age } \\
\text { (year) }\end{array}$ & $\begin{array}{c}\text { MAP } \\
(\mathrm{mmHg})\end{array}$ & $\begin{array}{l}\text { Heart Rate } \\
\text { (beats/min) }\end{array}$ & $\begin{array}{c}\mathrm{Na}^{+} \\
(\mathrm{mmol})\end{array}$ & $\begin{array}{c}\mathrm{K}^{+} \\
(\mathrm{mmol})\end{array}$ & $\begin{array}{c}\mathrm{Cl}^{-} \\
(\mathrm{mmol})\end{array}$ & $\begin{array}{c}\text { Protein } \\
(\mathrm{g} / \mathrm{l})\end{array}$ & $\begin{array}{c}\text { Glucose } \\
\text { (mmol) }\end{array}$ & $\begin{array}{c}\text { Urea } \\
\text { (mmol) }\end{array}$ & $\begin{array}{c}\text { WBC } \\
(1000 / \mu l)\end{array}$ & $\overline{P \text { PCV (\%) }}$ \\
\hline "Normal & "--- & $7.5-10.5$ & 60-100 & 138-142 & 3.5-4.9 & $100-110$ & 65-145 & 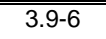 & 1.6- & 3.6-10 & $36-54$ \\
\hline Range & & & & & & & & & 8.33 & & \\
\hline Pregnant & $\begin{array}{c}27.09 \\
\pm 6.62\end{array}$ & $\begin{array}{c}13.27 \\
\pm 2.26\end{array}$ & $\begin{array}{l}109.45 \\
\pm 22.6\end{array}$ & $\begin{array}{r}140 \\
\pm 4.41\end{array}$ & $\begin{array}{c}4.12 \\
\pm 1.3\end{array}$ & $\begin{array}{l}102.18 \\
\pm 3.32\end{array}$ & $\begin{array}{c}68 \\
\pm 8.26\end{array}$ & $\begin{array}{c}9.67 \\
\pm 6.6\end{array}$ & $\begin{array}{r}4.87 \\
\pm 1.11\end{array}$ & $\begin{array}{c}13.73 \\
\pm 3.49\end{array}$ & $\begin{array}{c}34.67 \\
\pm 5.85\end{array}$ \\
\hline $\begin{array}{c}\text { Non } \\
\text { Pregnant }\end{array}$ & $\begin{array}{l}30.25 \\
\pm 9.56\end{array}$ & $\begin{array}{l}11.31 \\
\pm 2.14\end{array}$ & $\begin{array}{l}104.12 \\
\pm 19.77\end{array}$ & $\begin{array}{c}142.87 \\
\pm 3.31\end{array}$ & $\begin{array}{c}4.39 \\
\pm 0.48\end{array}$ & $\begin{array}{l}102.5 \\
\pm 2.78\end{array}$ & $\begin{array}{l}73.25 \\
\pm 6.65\end{array}$ & $\begin{array}{c}8.62 \\
\pm 2.76\end{array}$ & $\begin{array}{c}6.96 \\
\pm 1.96\end{array}$ & $\begin{array}{l}16.1 \\
\pm 4.3\end{array}$ & $\begin{array}{l}40.75 \\
\pm 6.54\end{array}$ \\
\hline$p$ & --- & $0.08 \mathrm{~A}$ & $0.61 \mathrm{~A}$ & $0.15 \mathrm{~A}$ & $0.6 \mathrm{~A}$ & $0.83 \mathrm{~A}$ & $0.17 \mathrm{~A}$ & $0.69 \mathrm{~A}$ & $0.01 * A$ & $0.21 \mathrm{~A}$ & $0.03^{*} \mathrm{~K}$ \\
\hline Multipara & $\begin{array}{l}25.14 \\
\pm 6.89 \\
\end{array}$ & $\begin{array}{l}13.86 \\
\pm 2.03 \\
\end{array}$ & $\begin{array}{l}110.86 \\
\pm 28.37 \\
\end{array}$ & $\begin{array}{c}139.86 \\
\pm 5.37 \\
\end{array}$ & $\begin{array}{c}4.23 \\
\pm 1.69 \\
\end{array}$ & $\begin{array}{c}101.71 \\
\pm 2.36 \\
\end{array}$ & $\begin{array}{l}69.71 \\
\pm 7.65 \\
\end{array}$ & $\begin{array}{c}11.4 \\
\pm 8.29 \\
\end{array}$ & $\begin{array}{l}4.47 \\
\pm 1.3 \\
\end{array}$ & $\begin{array}{l}14.41 \\
\pm 3.55 \\
\end{array}$ & $\begin{array}{l}33.73 \\
\pm 6.91 \\
\end{array}$ \\
\hline Nullipara & $\begin{array}{c}30.5 \\
\pm 6.45 \\
---\end{array}$ & $\begin{array}{c}12.25 \\
\pm 2.87 \\
0.3 \mathrm{~A}\end{array}$ & $\begin{array}{c}107 \\
\pm 15.79 \\
0.81 \mathrm{~A}\end{array}$ & $\begin{array}{c}140.25 \\
\pm 3.68 \\
0.9 \mathrm{~A}\end{array}$ & $\begin{array}{c}3.92 \\
\pm 0.62 \\
0.74 \mathrm{~A}\end{array}$ & $\begin{array}{c}103 \\
\pm 5.29 \\
0.58 \mathrm{~A}\end{array}$ & $\begin{array}{c}65 \\
\pm 10.68 \\
0.41 \mathrm{~A}\end{array}$ & $\begin{array}{c}6.64 \\
\pm 1.83 \\
0.3 \mathrm{~A}\end{array}$ & $\begin{array}{c}5.57 \\
\pm 0.3 \\
0.13 \mathrm{~A}\end{array}$ & $\begin{array}{l}12.52 \\
\pm 4.05 \\
0.44 \mathrm{~A}\end{array}$ & $\begin{array}{l}36.32 \\
\pm 4.93 \\
0.53 \mathrm{~A}\end{array}$ \\
\hline $1^{\text {st }}$ Trimester & $\begin{array}{l}24.25 \\
\pm 2.63 \\
\end{array}$ & $\begin{array}{l}13.25 \\
\pm 2.63 \\
\end{array}$ & $\begin{array}{c}126.5 \\
\pm 15.78 \\
\end{array}$ & $\begin{array}{l}139.5 \\
\pm 1.91 \\
\end{array}$ & $\begin{array}{c}4.68 \\
\pm 2.18 \\
\end{array}$ & $\begin{array}{l}100.5 \\
\pm 1.73 \\
\end{array}$ & $\begin{array}{l}67.25 \\
\pm 8.99 \\
\end{array}$ & $\begin{array}{c}14.45 \\
\pm 10.23 \\
\end{array}$ & $\begin{array}{c}5.4 \\
\pm 0.82\end{array}$ & $\begin{array}{l}11.62 \\
\pm 4.81\end{array}$ & $\begin{array}{c}36.8 \\
\pm 4.93\end{array}$ \\
\hline $2^{\text {nd }}$ Trimester & $\begin{array}{l}32 \\
\pm 7 \\
\end{array}$ & $\begin{array}{c}12 \\
\pm 3.46 \\
\end{array}$ & $\begin{array}{l}102.67 \\
\pm 16.16 \\
\end{array}$ & $\begin{array}{c}140.33 \\
\pm 4.51 \\
\end{array}$ & $\begin{array}{c}3.9 \\
\pm 0.62 \\
\end{array}$ & $\begin{array}{c}103.33 \\
\pm 6.11 \\
\end{array}$ & $\begin{array}{l}70.33 \\
\pm 5.03 \\
\end{array}$ & $\begin{array}{l}8.08 \\
\pm 2.7 \\
\end{array}$ & $\begin{array}{c}4.83 \\
\pm 1.15 \\
\end{array}$ & $\begin{array}{l}16.17 \\
\pm 2.33 \\
\end{array}$ & $\begin{array}{l}36.37 \\
\pm 6.52 \\
\end{array}$ \\
\hline $3^{\text {rd }}$ Trimester & $\begin{array}{r}26.25 \\
\pm 9.25 \\
\end{array}$ & $\begin{array}{l}14.25 \\
\pm 0.96 \\
\end{array}$ & $\begin{array}{c}97.5 \\
\pm 28.72 \\
\end{array}$ & $\begin{array}{c}140.25 \\
\pm 7.32 \\
\end{array}$ & $\begin{array}{c}3.72 \\
\pm 0.69 \\
\end{array}$ & $\begin{array}{c}103 \\
\pm 2.58 \\
\end{array}$ & $\begin{array}{c}67 \\
\pm 12.03 \\
\end{array}$ & $\begin{array}{r}6.82 \\
\pm 1.46 \\
\end{array}$ & $\begin{array}{c}4.37 \\
\pm 1.49 \\
\end{array}$ & $\begin{array}{c}14 \\
\pm 2.47 \\
\end{array}$ & $\begin{array}{r}31.27 \\
\pm 6.97 \\
\end{array}$ \\
\hline$p$ & --- & $0.88 \mathrm{~A}$ & $0.53 \mathrm{~A}$ & $0.97 \mathrm{~A}$ & $0.63 \mathrm{~A}$ & $0.53 \mathrm{~A}$ & $0.88 \mathrm{~A}$ & $0.22 \mathrm{~A}$ & $0.51 \mathrm{~A}$ & $0.29 \mathrm{~A}$ & $0.42 \mathrm{~A}$ \\
\hline Class I & $\begin{array}{c}21.8 \\
\pm 1.09\end{array}$ & $\begin{array}{c}14.2 \\
\pm 1.92\end{array}$ & $\begin{array}{c}115.2 \\
\pm 30.42\end{array}$ & $\begin{array}{l}139.2 \\
\pm 5.93\end{array}$ & $\begin{array}{c}3.54 \\
\pm 0.82\end{array}$ & $\begin{array}{c}102 \\
\pm 2.74\end{array}$ & $\begin{array}{c}67.6 \\
\pm 8.26\end{array}$ & $\begin{array}{l}10.16 \\
\pm 8.15\end{array}$ & $\begin{array}{c}4.5 \\
\pm 1.45\end{array}$ & $\begin{array}{l}14.44 \\
\pm 3.61\end{array}$ & $\begin{array}{l}31.24 \\
\pm 6.06\end{array}$ \\
\hline Class II & $\begin{array}{r}27.25 \\
\pm 1.26 \\
\end{array}$ & $\begin{array}{c}12.5 \\
\pm 2.64 \\
\end{array}$ & $\begin{array}{l}112 \\
\pm 16 \\
\end{array}$ & $\begin{array}{l}138.5 \\
\pm 1.91 \\
\end{array}$ & $\begin{array}{r}4.12 \\
\pm 0.51 \\
\end{array}$ & $\begin{array}{c}102 \\
\pm 5.42 \\
\end{array}$ & $\begin{array}{c}71.25 \\
\pm 4.5 \\
\end{array}$ & $\begin{array}{l}10.64 \\
\pm 7.78 \\
\end{array}$ & $\begin{array}{c}5.4 \\
\pm 0.11 \\
\end{array}$ & $\begin{array}{l}12.25 \\
\pm 4.14 \\
\end{array}$ & $\begin{array}{c}36.05 \\
\pm 4.9 \\
\end{array}$ \\
\hline Class III & $\begin{array}{c}40 \\
\pm 00 \\
\end{array}$ & $\begin{array}{c}12.5 \\
\pm 3.53 \\
\end{array}$ & $\begin{array}{c}90 \\
\pm 14.14 \\
\end{array}$ & $\begin{array}{l}145 \\
\pm 00 \\
\end{array}$ & $\begin{array}{c}3.55 \\
\pm 0.21 \\
\end{array}$ & $\begin{array}{c}103 \\
\pm 1.41 \\
\end{array}$ & $\begin{array}{c}62.5 \\
\pm 17.68 \\
\end{array}$ & $\begin{array}{c}8 \\
\pm 2.83 \\
\end{array}$ & $\begin{array}{c}4.75 \\
\pm 1.77 \\
\end{array}$ & $\begin{array}{l}14.9 \\
\pm 4.1 \\
\end{array}$ & $\begin{array}{c}40.5 \\
\pm 4.81 \\
\end{array}$ \\
\hline$p$ & --- & $0.55 \mathrm{~A}$ & $0.48 \mathrm{~A}$ & $0.25 \mathrm{~A}$ & $0.82 \mathrm{~A}$ & $0.93 \mathrm{~A}$ & $0.55 \mathrm{~A}$ & $0.92 \mathrm{~A}$ & $0.56 \mathrm{~A}$ & $0.64 \mathrm{~A}$ & $0.17 \mathrm{~A}$ \\
\hline
\end{tabular}

MAP: Medium arterial pressure; WBC: White blood cell count; $p$ : intergroup values; PCV: Packed cell volume;

Age classes: Class I: 20-23 years old; Class II: 26-29 years old and Class III: 40 years old;

*Significant difference when $\mathrm{p}<0.05$; A: Annova test; K: Kuruskal-Walis test;

Data were expressed as mean \pm SD (standard deviation).

\section{REFERENCES}

1 ABDEL RAHEIM MA., MEKI M., HOSSAM EL DIN M. O A bradykinin potentiating fraction isolated from the venom of Egyptian scorpion Buthus occitanus induced prostaglandin biosynthesis in female guinea pig. Comp. Biochem. Physiol. 1997, 116C, 183-9.

2 ANDRADE MV., CARMEZ MP., ABREU EM., DOLNIKOFF M., OMAR ED., VELASCO ID., CUNHA-MELO JR. Lung compliance, plasma electrolyte levels and acid-base balance are affected by scorpion envenomation in anesthetized rats under mechanical ventilation. Comp. Physiol. C. Toxicol. Pharmacol., 2004, 138, 97-104. 
3 BYTAUTIERE E., VEDERNIKOV YP., SAADE GR., ROMERO R., GARFEILD RE. Degranulation of the uterine mast cell modifies contractility of isolated myometrium from pregnant women. Am. J. Obstet. Gynecol; 2004, 191, 170510.

4 CHANG A., WOOD C. Fetal acid-base balance. Interdependence of maternal and fetal $\mathrm{PCO}_{2}$ and bicarbonate concentration. Am. J. Obstet. Gynecol., 1976, $125,61-4$

5 DEVAUX C., JOUIRO B., KRIFI MN., CLOT- FAYBESE O., EL AYEB M., ROCHAT H. Quantitative variability in the biodistribution and in toxicokinetic studies of the three main alpha toxins from the Androctonus australis hector scorpion venom. Toxicon; 2004, 34, 661-9.

6 D'SUZE G., MONCADA S., GONZALEZ C., SERVICK C., AGUILAR V., AAGON A. Relationship between plasmatic levels of various cytokines, tumor necrosis factor, enzymes, glucose and venom concentration following Tityus serrulatus scorpion sting. Toxicon, 2003, 41, 367-75.

7 ELSTON DM. Life-threatening stings, bites, infestations, and parasitic diseases; Clin. Dermatol., 2003, 41, 367-75.

8 FUKUHARA YDM., DELLALIBERA- JOVILIANO R., CUNHA FQC., DONADI EA. Increased plasma levels of IL-1 beta, IL-6, IL-8, IL-10 and TNF-alpha in patients moderately or severely envenomed by Tityus serrulatus scorpion sting. Toxicon, 2003, 41, 49-55.

9 FUKUHARA YDM., DELLALIBERA- JOVILIANO R., CUNHA FQC., DONADI EA. The kinin system in the envenomation caused by the Tityus serrulatus scorpion sting. Toxicol. Appl. Pharmacol., 2004, 196, 390-5.

10 ISMAIL M. The scorpion envenoming syndrome. Toxicon, 1995, 33, 825-58.

11 JEDDI H.M. Les scorpions tunisiens. In JeDDI H.M., HAMIDA C., DAMAK J., JEDDI M., AYADI N: Piqure par scorpion chez Thomme en Tunisie: etude epidemiologique. Sfax: COOPI Sfax, 1989, 17-22.

12 KALAPOTHAKIS E., CHAVEZ- OLORTEGUI C. Venom variability among several Tityus serrulatus specimens. Toxicon, 1997, 35, 1523-9. 
13 KRIFI MN; KHARRAT H, ZGHAL K, ABDOULI M, ABROUG F, BOUCHOUCHA S, DELLAGI K, EL AYEB M. Development of an ELISA for the detection of scorpion venoms in sera of humans envenomed by Androctonus australis garzonï (AAG) and Buthus occitanus tunetanus (BOT): correlation with clinical severity of envenoming in Tunisia; Toxicon, 1998, 26, 277-88.

14 KRIFI MN., MARRAKCHI N., EL AYEB M., DELLAGI K. Effect of some variables on the in vivo determination of scorpion and viper venom toxicities. Biologicals, 1998, 36, 887-900.

15 KUBOW S., KOSKI KG. Maternal dietary glucose-lipid interactions modulate embryological development in vivo and in embryo culture. Biol. Reprod., 1995, 52, 145-55.

16 LANGLEY RL. A review of venomous animal bites and stings in pregnant patients. Wilderness Environ. Med., 2004, 15, 207-15.

17 MAZZEI DE DAVILA CA., DAVILA DF., DONIS JH., DE BELLABABA GA., VILLARREAL V., BARBOZA JS. Sympathetic nervous system activation, antivenom administration and cardiovascular manifestations of scorpion envenomation. Toxicon, 2002, 40, 1339-46.

18 MENDONÇA M., DALUZ MM., FREIRE-MAIA L., CUNHA-MELO JR. Effect of scorpion toxin from Tityus serrulatus on the contraction of the isolated rat uterus. Toxicon, 1995, 33, 355-61.

19 MEREI ZA., IBRAHIM SA. Stimulation of the rat uterus by the venom of the scorpion Leiurus quinquestriatus. Toxicon; 1979, 13, 251-8.

20 MILSOM I., HEDNER N., MANNEHEIMER C. A comparative study of the effect of high-intensity transcutaneous nerve stimulation and oral naproxen on intrauterine pressure and menstrual pain in patients with primary dysmenorrhea. Am. J. Obstet. Gynecol. 1994, 170, 123-9.

21 NASSAR Y., ABU SINNA G., RAHIM S.A. Effect of a bradykinin-potentiating fraction, from the venom of the Egyptian scorpion Buthus occitanus, on the ovaries and endothelium of mice. Toxicon, 1990, 28, 524-34.

22 OSMAN OH., ISMAIL M., EL ASMAR MF., IBRAHIM SA. Effect on the rat uterus of the venom from the scorpion Leiurus quinquestriatus. Toxicon, 1972, 10, 363-6. 
23 PARANT O., CLONET-DLONNAY M., CONNAN L., DUCLUSAND A., CHALE J., FOURNIE A. Metrorrhagia during the second trimester of pregnancy: obstetrical and perinatal outcome. A retrospective study including 85 cases. J. Gynecol. Obstet. Biol. Reprod., 2000, 29, 66-72.

24 PARIMI PS., CRONIGER CM., LEAHY P., HANSON RH., KALHAN SC. Effect of reduced maternal inspired oxygen on hepatic glucose metabolism in the rat fetus. Pediatr. Res., 2003, 53, 325-32.

25 PELTIER MR. Immunology of term and preterm labor. Reprod. Biol. Endocrinol., 2003, 1, 122 (http://www.rbej.com/).

26 SADOWSKY DW., HALUSKA GJ. Indomethacin blocks interleukin 1 betainduced myometrial contractions in pregnant rhesus monkeys. Am. J. Obstet. Gynecol., 2000, 183, 173-80.

27 SCALZONE JM., WELLS SL. Latrodectus mactans (black widow spider) envenomation: an unusual cause for abnormal pain in pregnancy. Obstet. Gynecol., 1994, 83, 830-1.

28 SHAH S., MILLER PR., MEREDITH JW., CHANG MC. Elevated admission white blood cell count in pregnant trauma patients: an indication of ongoing placental abruption. Am. Surg., 2002, 68, 644-7.

29 SHERMAN RP., GROLL JM., GONZALEZ DI., AERTS MA. Black widow spider (Lactrodectus mactans) envenomation in a term pregnancy. Curr. Surg., 2000, 57, 346-8.

30 SOUDANI N., GHARBI-CHIHI J., SRAIRI-ABID N., YAZIDI CM., PLANELLS R., MARGOTAT A., TORRESSANI J., EL AYEB M. Isolation and molecular characterization of LVP1 lipolysis activating peptide from scorpion Buthus occitanus tunetanus. Biochim. Biophys. Acta., 2005, 1747, 47-56. (http://www.sciencedirect.com/science/journal/00063002).

31 WOODS LL., THRONBURG KL., FABER JJ. Transplacental gradients in the Guinea pig. Am. J. Physiol. 1978, 235, H 200-7.

32 ZEGHAL K., SAHNOUN Z., GUINOT M., GIUDICELLI JF. Characterization and mechanisms of the cardiovascular and haemodynamic alterations induced by scorpion venom in rats. Fund. Clin. Pharmacol. 200, 14, 351-61. 